when the American Foundation for AIDS Research started to take an interest that Hütter engaged well-recognized names, and NEJM eventually accepted his paper.

More importantly, the Berlin patients' cases have inspired new scientific ideas. We still do not know what led to either cure. Many researchers think that Christian is probably an 'elite controller', one of the $1 \%$ of people with HIV whose genetic make-up gives them an effective immune response to the virus. So he may have controlled his virus at the outset. However, his case raised the possibility that very early treatment could reduce or prevent the formation of long-lived reservoirs, as was demonstrated last year in 14 people with HIV in the VISCONTI (Viro-Immunological Sustained Control after Treatment Interruption) study in France. Christian's case also revealed the importance of the role of the T-cell immune response in controlling the virus - a major stimulus to HIV vaccine development.

With Brown, was the cure a reaction of the donor marrow against his immune system, a process called graft-versus-host disease? Was it down to the intensive conditioning treatment he received before the transplant? Or was it that the donor marrow was CCR5-negative? A promising treatment based on eliminating CCR5 using gene therapy was developed in a mouse model by a team including Holt, and is now being tested in clinical trials.

I enjoyed the book but I do have quibbles. Holt's discussions can be dense and detailed. Her interviews, which tend to focus on US-based leaders in HIV science, make it seem as if little discovery was being led from elsewhere, even though there have been significant contributions from many other countries, not least France, where the initial discovery of the virus was made. This is an important book, nevertheless.

Although the Berlin patients' case reports are anecdotes, they convinced a sceptical scientific world that a cure was possible, and inspired lines of enquiry and investment. On World AIDS Day 2013, US President Barack Obama announced that his administration would give an extra US\$100 million to research towards a cure. Most importantly, the cases belong to a significant handful that is giving hope to millions of people living with HIV.

Sharon Lewin is a clinician scientist working on approaches to finding a cure for HIV. She is professor and head of the department of infectious disease at Alfred Health and Monash University in Melbourne, Australia, and co-head of the Centre for Biomedical Research at the Burnet Institute in Melbourne. e-mail:sharon.lewin@monash.edu

\title{
Inside utter strangeness
}

\section{David Seery applauds a primer on the incongruous world of quantum mechanics.}

I is all too easy to compound the perplexities of quantum mechanics. Monographs are often weighed down by abstruse mathematics; other treatments struggle to separate physics from formalism. The problem is to communicate the subject's intrinsic strangeness without burdensome mathematical demands - but also without resorting to gee-whiz platitudes that assert, but do not explain, the incongruities between the quantum world and everyday experience.

Leonard Susskind and Art Friedman's Quantum Mechanics is the second entry in Susskind's Theoretical Minimum series, based on an Internet course of the same name and designed to satisfy a mathematically literate audience looking for more detail than can be found in a popular-science offering. The authors set their aims at the outset: "the goal", they say, "is not to hide the utter strangeness of quantum logic but to bring it out into the light of day".

To achieve this, Susskind, a physicist, and Friedman, a data engineer, eschew the analogies and qualitative descriptions typically used to explain quantum mechanics to a lay audience. Instead, readers are expected to invest time in getting to grips with mathematical concepts such as complex numbers, vector spaces, linear operators and tensor products, all of which are deftly explained in a series of interludes. In return, the authors offer self-contained chapters that vividly articulate aspects of the theory: the space of states, time evolution, uncertainty, entanglement, particle dynamics and so on, leading eventually to the harmonic oscillator, a quantized mechanical spring. This is the most important of all simple quantum systems because quantum field theory, the discipline's description of the forces in nature, interprets particles as harmonic oscillators.

The selection of concepts is close to what would be found in a typical undergraduate course. It steers clear of the philosophical morass surrounding interpretation and focuses on how quantum mechanics is used and understood today, rather than its development or the human stories behind it. This is a book about physics, not history.

The writing is fresh and immediate, with plenty of detail packaged into the smooth narrative. It will not teach anyone to carry out complex calculations, and it may leave the reader with questions that can be resolved

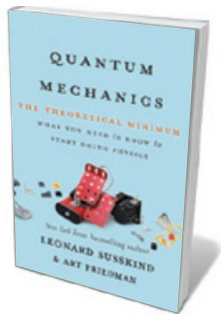

only by digging into more conventional textbooks. But on their own terms, I found Susskind and Friedman's explanations crisp and satisfying. The chapters on entanglement - in which particles interact in such a way that they cannot be described independently - are especially clear, taking pains to draw out the distinction between quantum entanglement and correlation. In either case, we can predict something

Mechanics:

The Theoretical Minimum: What You Need to Know to Start Doing

Physics

LEONARD SUSSKIND AND ART FRIEDMAN Basic Books/Allen Lane: 2014. about the relationship between measure-
ments in different parts of a composite system. The puzzling feature of entanglement is that, although we may know everything about the state, it might be impossible to predict anything about the outcome of these measurements individually.

Susskind and Friedman's choices will suit their intended readership, but might make the book less attractive to others. Those with the determination to navigate the complexities of Hermitian operators, normalizable wavefunctions or creation-annihilation operators will struggle to find a book that serves them better. Secondary-school pupils, however, may find the more detailed passages too demanding; university students might find that the early chapters proceed slowly, and that overall the book is too light on detail to serve as a principal text.

Still, I maintain a clear recollection of the bewilderment with which I struggled through my own university quantummechanics courses. For students in a similar position, trying to draw together the fragments of formalism into a clear conceptual whole, Susskind and Friedman's persuasive overview - and their insistence on explaining, with sharp mathematical detail, exactly what it is that is strange about quantum mechanics - may be just what is needed.

David Seery is reader in mathematics and physics at the University of Sussex in Brighton, UK.

e-mail:d.seery@sussex.ac.uk 